was used as newly diagnosed maintenance treatment in 25 (58.1\%) patients, as treatment for $\geq 3$ line recurrence treatment in $14(32.6 \%)$ patients, and as platinum-sensitive recurrent maintenance treatment in $4(9.3 \%)$ patients. Overall, 28 (65.1\%) patients experienced $\geq 1$ grade hematologic AEs, which included leukopenia (37.2\%), anemia (34.9\%) and thrombocytopenia (39.5\%). Only $10 \quad(23.3 \%)$ patients had grade 3/4 AEs including leukopenia (9.3\%), anemia (7.0\%) and thrombocytopenia (11.6\%). Until last follow up, the median time for the occurrence of leukopenia, anemia and thrombocytopenia were 30 (range: 7, 162), 34 (range: 7, 108) and 20 (range: 13, 180) days, respectively. No deaths were reported. Of those patients who experienced AEs during treatment, the dose was reduced in $4(14.3 \%)$ patients, and treatment was interrupted in $9(32.1 \%)$ patients. Additional recombinant human granulocyte colony stimulating factor $(n=5,17.9 \%)$, erythrocyte $(n=2,7.1 \%)$ and recombinant human thrombopoietin $(n=5,17.9 \%)$ were provided for treating the AEs. After intervention, $8(18.6 \%)$ patients restart the treatment and only $1(2.3 \%)$ patient discontinued the treatment.

Conclusion The incidence of hematologic AEs in real-world experience was lower than reported by niraparib $300 \mathrm{mg} /$ day in ENGOT-OV16/NOVA trial. In addition to maintenance treatment in the first line, the patients in platinum-sensitive recurrence treatment and later line treatment might benefit from ISD niraparib.

Disclosures The authors declare that they have no competing interests.

\section{SIGNALING PATHWAYS RELATED WITH ITGBL1 IN OVARIAN CANCER CELLS}

${ }^{1}$ Alexander J Cortez, ${ }^{2}$ Katarzyna A Kujawa, ${ }^{3}$ Agata M Wilk, ${ }^{3}$ Marcela K Krzempek, ${ }^{2}$ Joanna P Syrkis, ${ }^{2}$ Magdalena Olbryt, ${ }^{2}$ Katarzyna M Lisowska. 'Maria Sklodowska-Curie National Research Institute of Oncology, Gliwice Branch; Department of Biostatistics and Bioinformatics, Center for Translational Research and Molecular Biology of Cancer; ${ }^{2}$ Maria Sklodowska-Curie National Research Institute of Oncology, Gliwice Branch; Center for Translational Research and Molecular Biology of Cancer; ${ }^{3}$ Maria Sklodowska-Curie National Research Institute of Oncology, Gliwice Branch; Department of Biostatistics and Bioinformatics

\subsection{6/ijgc-2020-ESGO.135}

Introduction/Background Integrin beta-like 1 (ITGBL1) is a poorly characterized protein comprised of ten EGF-like repeats. Our previous studies suggested that higher ITGBL1 mRNA expression level in the tumor is related with shorter survival of ovarian cancer patients. ${ }^{1}{ }^{2}$ Subsequent functional in vitro studies revealed that ITGBL1 overexpression in ovarian cancer cells resulted in the altered adhesion, migration and invasiveness, while it had no effect on proliferation rate and the cell cycle. ITGBL1-overexpressing cells were significantly more resistant to cisplatin and paclitaxel, ${ }^{3}$ major drugs used in OC treatment. ${ }^{4}$ In the current study we analyzed gene expression profiles of ITGBL1-overexpressing and control ovarian cancer cells and investigated ITGBL1 influence on ovarian cancer cell signaling pathways.

Methodology ITGBL1 coding sequence was PCR-amplified from cDNA and cloned into pLNCX2 vector. Retroviral system was used to obtain two ovarian cancer cell lines: OAW42/ITGBL1(+) and SKOV3/ITGBL1(+) with overexpression of ITGBL1. Control cell lines were obtained by transduction with an empty vector. RNA was isolated from wild type,
ITGBL1-overexpressing and control cells. DNA microarray experiment was performed using GeneChip ${ }^{\text {тм }}$ Human Transcriptome Array 2.0 (Affymetrix, Santa Clara, CA, USA) according to the manufacturer's instructions. Bioinformatical analysis was carried out in $\mathrm{R}$ environment (version 3.5.3) with Bioconductor packages.

Results Using Principal Component Analysis, an unsupervised method of data analysis, we selected gene sets related to major sources of variability in our dataset. Then, by performing Gene Set Enrichment Analysis we found 76 and 146 significantly affected cellular signaling pathways (in OAW42 and SKOV3 cell line, respectively). Majority of them (22 and 44, respectively) were related to extracellular matrix structure and function, integrin signaling, focal adhesion, cell junction, cellular motility, ERBB2 and ERBB4 signaling, etc.

Conclusion Global gene expression analysis revealed that signaling pathways affected by ITGBL1 overexpression were mostly those related to extracellular matrix organization and function, integrin signaling, focal adhesion, cellular communication and motility. These results are concordant with functional changes observed in ITGBL1-overexpressing cells, like altered adhesiveness, enhanced motility and invasiveness. Overall, our results indicate that higher expression of ITGBL1 in ovarian cancer cells is associated with features that may worsen clinical course of the disease.

A.J.Cortez was co-financed by the EU through the European Social Fund (grant-POWR.03.02.00-00-I029).

\section{REFERENCES}

1. Lisowska,et al.(2014),DOl:10.3389/fonc.2014.00006

2. Lisowska, et al.(2016),DOl:10.1007/s00432-016-2147-y

3. Cortez,et al.(2020),DOI: 10.3390/cancers12092676

4. Cortez,et al.(2020),DOI: 10.1007/s00280-017-3501-8

Disclosures Authors have nothing to disclose.

\section{FIBRONECTIN AND PERIOSTIN AS PROGNOSTIC MARKERS IN OVARIAN CANCER}

${ }^{1}$ Katarzyna A Kujawa, ${ }^{2}$ Ewa Zembala-Nożyńska, ${ }^{3}$ Alexander J Cortez, ${ }^{4}$ Jolanta Kupryjańczyk ${ }^{5}$ Katarzyna M Lisowska. 'Maria Sklodowska-Curie National Research Institute of Oncology, Gliwice Branch; Center for Translational Research and Molecular Biology of Cancer; ${ }^{2}$ Maria Sklodowska-Curie National Research Institute of Oncology, Gliwice Branch; Tumor Pathology Department: ${ }^{3}$ Maria Sklodowska-Curie National Research Institute of Oncology, Gliwice Branch; Department of Biostatistics and Bioinformatics, Center for Translational Research and Molecular Biology of Cancer; ${ }^{4}$ Maria Skłodowska-Curie Memorial Cancer Center and Institute of Oncology; Tumor Pathology Department; ${ }^{5}$ Maria Sklodowska-Curie National Research Institute of Oncology, Gliwice Branch; Center for Translational Research and Molecular Biology of Cancer

\subsection{6/ijgc-2020-ESG0.136}

Introduction/Background In our previous microarray study we identified a 96-gene prognostic signature associated with the shorter overall survival (OS) of ovarian cancer patients. ${ }^{1}$ Two genes from this signature, both coding for extracellular matrix proteins, were objects of the present study: FN1 and POSTN. We analyzed, by immunostaining, expression of encoded proteins in the independent set of ovarian cancer samples and evaluated its correlation with clinical, pathological, and molecular features.

Methodology Ovarian cancer samples from 108 patients were analyzed by immunochistochemistry using rabbit anti-human fibronectin polyclonal antibody (1:3000 dilution, A0245, Dako, Glostrup, USA) and rabbit anti-human periostin 